\title{
FATORES QUE INTERFEREM NO PROGNÓSTICO DE DEAMBULAÇÃO NA PARALISIA CEREBRAL
}

\author{
ANGELA M. C. SOUZA* - IVAN FERRARETTO **
}

\begin{abstract}
RESUMO - Com o objetivo de estudar o prognóstico de deambulação em crianças com paralisia cerebral, foi feito estudo retrospectivo de 100 crianças em tratamento na Associação de Assistência à Criança Defeituosa, no período de 1979 a 1988 . Foram analisados os seguintes parâmetros, correlacionando-os à capacidade de adquirir ou não a criança a deambulação: (1) grau de comprometimento motor; (2) idade de aquisição do equilíbrio sentado; (3) função de membros superiores; (4) grau de retardo mental; (5) presença de reflexos primitivos. Os resultados de nosso estudo sugerem que são fatores indicativos de mau prognóstico de deambulação: (1) gravidade do quadro motor; (2) aquisição da postura sentada após os 3 anos; (3) função precária de membros superiores; (4) retardo mental grave associado a grave deficiência física. A avaliação clínica de reflexos primitivos das crianças do nosso estudo não revelou relação direta com o prognóstico de deambulação.
\end{abstract}

PALAVRAS CHAVE: paralisia cerebral, deambulação, prognóstico.

Clinical digns for the prognosis of walking in cerebral palsy.

SUMMARY - With the objective of studying the prognosis for walking in children with cerebral palsy a retrospective survey of 100 children being treated at the Associação de Assistência à Criança Defeituosa was made during the period from 1979 to 1988 . The following parameters were analysed: (1) degree of motor dysfunction; (2) age of acquisition of sitting balance; (3) function of upper limbs; (4) degree of mental retardation; (5) presence of primitive reflexes. All the above parameters were analysed with respect to the capability of children acquiring or not walking. The results of our study show that the following factors are indicative of bad prognosis for walking: (1) severity of motor dysfunction; (2) acquisition of sitting posture after 3 years old; (3) precarius function of upper limbs; (4) severe mental retardation associated with severe physical deficiency. Clinical evaluation of primitive reflexes did not reveal direct relation to the walking prognosis.

KEY WORDS: cerebral palsy, walking, prognosis.

Após o diagnóstico de paralisia cerebral em uma criança, o profissional se depara com uma questão fundamental - Qual o prognóstico de deambulação? Com a finalidade de responder a essa pergunta correlacionamos dados de avaliação clinica - como: (1) quadro motor; (2) idade de aquisição do equilibrio sentado; (3) função de membros superiores; (4) grau de retardo mental; (5) presença de reflexos primitivos - e comparamos esses itens à capacidade ou nầo de vir a criança a adquirir deambulação.

\section{CASUíSTICA E MÉTODO}

Analisamos, retrospectivamente, 100 prontuários de crianças com paralisia cerebral (PC) em tratamento na AACD, no período entre 1979 e 1988. Todas as crianças, no exame clinico inicial, não tinham adquirido a deambulação. Estes pacientes foram submetidos a um pro-

Associação de Assistência à Criança Defeituosa (AACD): * Médica Coordenadora da Clínica de Paralisia Cerebral; ** Diretor Clínico e Cirurgião Chefe da AACD.

Dra. Angela M. C. Souza - AACD, Associação de Assistência à Criança Defeituosa - Av. Prof. Ascendino Reis 724 - 040Z7 São Paulo SP - Brasil. 
grama de reabilitação e a testes de capacidade intelectual. O seguimento clínico foi em média de 4 anos (min.: 3 a. e máx. 5 a.). A média de idade no exame clínico inicial era 3 a. (min.: 1 a. e máx.: 13 a.). Em relação ao sexo, 60 crianças eram meninos e 40, meninas. A distribuição diagnóstica dos 100 casos é apresentada na Tabela 1.

Tabela 1 - Distribuição diagnóstica.

\begin{tabular}{|c|c|}
\hline Diagnóstico & No de pacientes \\
\hline Tetraparesia espástica & 18 \\
\hline Tetraparesia mista (espástica-atetóide) & 7 \\
\hline Tetraparesia coreoatetoide & 11 \\
\hline Triparesia espástica & 13 \\
\hline Triparesia mista & 1 \\
\hline Diparesia espástica & 36 \\
\hline Diparesia mista & 3 \\
\hline Paraparesia espástica & 8 \\
\hline Paraparesia mista (espástica-atáxica) & 1 \\
\hline Hemiparesia espástica & 2 \\
\hline Total & 100 \\
\hline
\end{tabular}

Analisamos os seguintes parâmetros correlacionando-os à aquisição ou não de deambulação e submetendo os dados à análise estatística: (1) grau de comprometimento motor; (2) idade de laquisição da postura sentada; (3) função de membros superiores; (4) avaliação intelectual; (5) presença de reflexos primitivos (Moro, RTCA, RTCS, Reação Positiva de Apoio, ausência da Reação de Paraquedas). Analisamos a presença de reflexos primitivos somente no grupo com PC tipo diparesia espástica, pois sabemos que os reflexos primitivos estão presentes com maior frequência no grupo de maior gravidade motora, o que poderia falsear o resultado das nossas observações. Consideramos a função manual: boa ou razoável quando a criança tinha preensão ativa de um objeto, conseguindo soltá-lo voluntariamente; precária, quando o paciente não conseguia a preensão e o soltar ativo de objetos. A função intelectual foi considerada de acordo com a Classificação da OMS.

\section{RESULTADOS}

Grau de comprometimento motor e aquisição de deambulação - Observamos a seguinte pruporção: tetraparéticos $2,77 \%$ adquiriram deambulação; triparéticos $38,47 \%$; diparéticos $60,55 \%$; paraparéticos 100\%; hemiparéticos $100 \%$.

Idade de aquisição do equilíbrio sentado e a aquisição de deambulação - Obtivemos, para correlação, os resultados anotados na Tabela 2.

Tabela \& - Idade de aquisiçāo da postura sentada $\times$ deambulação.

\begin{tabular}{|c|c|c|c|c|}
\hline \multirow[t]{2}{*}{ Idade sentar } & \multicolumn{2}{|c|}{ Deambuladores } & \multicolumn{2}{|c|}{ Não deambuladores } \\
\hline & No & $\%$ & $\mathrm{~N}^{\circ}$ & $\%$ \\
\hline Antes de 3 anos & 34 & 91,8 & 7 & 11 \\
\hline Depois de 3 anos & 3 & 8,1 & 52 & 89 \\
\hline
\end{tabular}

$\chi^{2}=59,52(p<0,001)$. 
Função dos membros superiores e aquisição de deambulação - Observamos por correlação que 35 (74\%) dos que adquiriram deambulação tinham os membros superiores com boa função; ao contrário, $48(76,6 \%)$ dos pacientes que não andaram tinham os membros superiores com função precária.

Avaliação intelectual e a possibilidade de adquirir deambulação - A análise dos dados mostrou que $30(85,8 \%)$ dos pacientes que andaram tinham deficiência mental de leve a moderada e apenas $5(14,2 \%)$ tinham deficiência mental grave.

Presença de reflexos primitivos - Foi estudada no grupo de PC, tipo diparesia espástica, estando os resultados na Tabelia 3.

Tabela 3 - Presença de reflexos primitivos em $P C$ diparéticos espásticos.

\begin{tabular}{|c|c|c|c|c|}
\hline \multirow[b]{2}{*}{ Reflexos primitivos } & \multicolumn{2}{|c|}{ Deambuladores } & \multicolumn{2}{|c|}{ Não deambuladores } \\
\hline & $\begin{array}{r}\mathrm{N}: \\
15\end{array}$ & $\begin{array}{r}\% \\
100\end{array}$ & $\begin{array}{l}\text { Ne } \\
11\end{array}$ & $\begin{array}{r}\% \\
100\end{array}$ \\
\hline Moro & 3 & 20 & 5 & 45 \\
\hline RTCA & 5 & 33 & 5 & 45 \\
\hline RTCS & 3 & 20 & 1 & 9 \\
\hline R. posit. apoio & 11 & 73 & 8 & 72 \\
\hline Aus. paraquedas & 4 & 26 & $y$ & 63 \\
\hline
\end{tabular}

\section{COMENTARIOS}

$\dot{E}$ clássica a afirmação de que quanto mais grave e global for o acometimento motor, menores são as chances de a criança com PC adquirir deambulação 1-10.

A correlação da presença de reflexos primtivos com a deambulação foi feita por Bleck 2 que referiu a presença de reflexos primitivos como dado altamente signıficativo para a não aquisição de deambulação. Em nossa população de $\mathrm{PC}$, tipo diparesia espástica, verificamos que a presença de reflexos primtivos, como sinal isolado, não justifica a indicação de mau prognóstico de marcha. Acreditamos que a diferença de conclusões foi dada pela amostra de pacientes: na série de Bleck, dos 17 pacientes que não andaram 16 eram $P C$ tetraparéticos, que não teriam chance de adquirir deambulação, devido à gravidade do quadro motor.

A relação da idade de aquisição da postura sentåda e aquisição da deambulação é muito significativa nos estudos feitos por Molnar e Gordon 8 , afirmando eles que, se a postura sentada não for adquirida antes dos 2 aos 3 anos, o prognóstico de deambulação independente será ruim. Nosso estudo comprovou essas afirmações (Tabela 2).

Quanto mais grave for o acometimento dos membros superiores do paciente, mais global será o seu comprometimento motor. Este item merece atenção nos casos em que a criança irá necessitar de apoio para a marcha, como andadores ou muletas e, portanto, de membros superiores funcionais.

Assinalamos que nossa população de pacientes com deficiência mental grave também tinha grave comprometimento motor que os impediria de andar, independente do retardo mental. Consideramos, portanto, que este parâmetro deve ser melhor analisado e este tema é motivo de outro estudo, em fase de conclusão, na Clínica de Paralisia Cerebral da AACD.

Em conclusão, os resultados de nosso estudo sugerem que são fatores indicativos de mau prognóstico de deambulação: (1) gravidade do quadro motor; (2) retardo de aquisição do equilíbrio sentádo após os 3 anos; (3) função precária de membros superiores; (4) retardo mental grave associado a deficiência física grave. A avaliação clínica de reflexos primitivos das crianças com $\mathrm{PC}$, do nosso estudo, não revelou relação direta com o prognóstico de deambulação.

Agradecimentos - Ao Dr. Neil Ferreina Novo, Professor Adjunto, Disciplina Bioestatística, Escola Paulista de Medicina. 


\section{REFERENCIAS}

1. Balf CL, Ingram TTS. Problems in the classification of cerebral palsy in childhood. $\mathrm{Br}$ Med J 1955, 16:163-166.

2. Bleck EE. Locomotor prognosis in cerebral palsy. Dev Med Child Neurol 1975, 17:18-25.

3. Capute JA. Primitive reflex profile.' Physical Ther 1978, 9:1061-1065.

4. Ezawa K, Nakajima K, Mori H. Ambulation prognosis in very severely multi-handicapped children. Bull Tokyo Metropolitan Rehabilitation Center 1980, 11-16.

5. Harris SR. Early neuromotor predictors of cerebral palsy in low-birthweight infants. Dev Med Child Neurol 1987, 29:508-519.

6. Hoffer M. Feiwell E, Perry R, Perry J, Bonnett C. Functional ambulation in patients with myelomeningocele. J Bone Joint Surg 1973, 55A:137-148.

7. Molnar G. Cerebral palsy: prognosis and how to judge it. Pediatric Ann 1979, 8:596-605.

8. Molnar G. Gordon S. Cerebral palsy: predictive value of selected clinical signs for early prognostication of motor function.' Arch Phys Med Rehab 1976, 57:153-158.

9. Paine RS. The evolution of infantile postural reflexes in the presence of chronic brain syndromes. Dev Med Child Neurol 1964, 6:345-361.

10. Palmer BF, Shafiro BK, Watchtel RC, Capute JA. Primitive reflex profile. In Thompson GH, Rubin IL, Bilenker RM (eds): Comprehensive Management of Cerebral Palsy. New York: Grune \& Stratton 1983, p 171-179. 